\title{
Avaliação radiográfica dos pacientes submetidos a fixação percutânea com parafuso maciço para tratamento de fraturas da extremidade distal do radio*
}

\section{Radiographic Evaluation of Patients Submitted to Percutaneous Fixation With Solid Screw for Distal Radius Fracture Treatment}

\author{
Gonzalo Guamán Gaibor ${ }^{10}$ Leonardo Dalla Giacomassa Rocha Thomaz ${ }^{1}$ Cristian Stein Borges ${ }^{3(0)}$ \\ Paulo Henrique Ruschel ${ }^{10}$ Milton Bernardes Pignataro ${ }^{10}$
${ }^{1}$ Serviço de Ortopedia e Traumatologia, Santa Casa de Porto Alegre, Porto Alegre, RS, Brasil
Rev Bras Ortop 2020;55(5):605-611.
Endereço para correspondência Leonardo Dalla Giacomassa Rocha Thomaz, MD, Santa Casa de Misericórdia de Porto Alegre, Av. Independência, 75, Independência, Porto Alegre, RS, 90035-072, Brasil (e-mail: leorochat@hotmail.com).

\section{Resumo \\ Palavras-chave \\ - fraturas do rádio \\ - procedimentos cirúrgicos minimamente invasivos \\ - radiologia}

Abstract
Objetivo Apresentar o resultado das avaliações radiográficas dos pacientes com fraturas extra-articulares do rádio distal submetidos a fixação percutânea com parafuso maciço de $3.5 \mathrm{~mm}$.

Métodos Série de casos, analítica, descritiva e retrospectiva de 16 pacientes com avaliação dos parâmetros radiográficos da técnica utilizada.

Resultados A média de idade da população estudada foi de 46,5 anos (25-60 anos), sendo $81,25 \%$ do sexo feminino. O tempo de espera até a cirurgia foi de 8,8 dias (4-14 dias). 0 tilt volar médio pré-procedimento foi $-7,41^{\circ}\left(-23,48^{\circ}-5,29^{\circ}\right.$, desvio padrão $\left.[D P] \pm 6,59^{\circ}\right)$. O tilt volar imediatamente após o procedimento cirúrgico foi de $5,93^{\circ}$ $\left(\mathrm{DP} \pm 6,23^{\circ}, p<0,001\right)$. Sem diferença estatística nos valores de tilt volar ao final de 6 meses de evolução. A altura radial pré-procedimento foi de $4,13 \mathrm{~mm}\left(-7,8^{\circ}-9,5^{\circ}\right.$, $\left.\mathrm{DP} \pm 5,06^{\circ}\right)$. Aumento estatisticamente significativo no pós-operatório imediato para $10,04 \mathrm{~mm}(p=0,002)$. Redução significativa ao final de 6 meses para $9,55 \mathrm{~mm}$ $(p=0,012)$. Atingimos $100 \%$ de consolidação com a técnica utilizada, com índice mínimo de complicações. Nenhum paciente apresentou infecção ou precisou ser reoperado.

Conclusão A técnica mostrou-se eficaz para o tratamento de fraturas extra-articulares da extremidade distal do rádio ao final de 6 meses, com baixo índice de complicações e parâmetros radiográficos aceitáveis e próximos dos valores anatômicos.

Objective The present paper aims to present results from radiographic evaluations of patients with extra-articular distal radius fractures submitted to percutaneous fixation with a $3.5 \mathrm{~mm}$ solid screw.

Trabalho desenvolvido no Serviço de Ortopedia e Traumatologia,

Santa Casa de Porto Alegre, Porto Alegre, RS, Brasil.

recebido

30 de Julho de 2019

aceito

12 de Novembro de 2019
DOI https://doi.org/

10.1055/s-0040-1702952. ISSN 0102-3616.
Copyright $\odot 2020$ by Sociedade Brasileira License terms de Ortopedia e Traumatologia. Published by Thieme Revinter Publicações Ltda, Rio de Janeiro, Brazil 


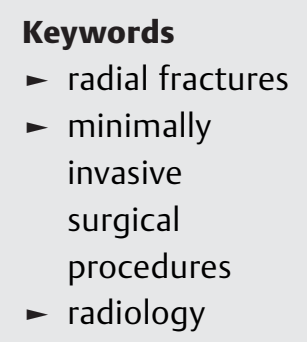

Methods Analytical, descriptive and retrospective case series of 16 patients with evaluation of the radiographic parameters.

Results The average age of the study population was 46.5 years old (25-60 years old); $81.25 \%$ of the sample was female. The average time until surgery was 8.8 days (4-14 days). The mean preprocedural volar tilt was $-7.41^{\circ}\left(-23.48^{\circ}-5.29^{\circ}\right.$, standard deviation $\left.[S D] \pm 6.59^{\circ}\right)$. The mean volar tilt immediately after surgery was $5.93^{\circ}$ $\left(\mathrm{SD} \pm 6.23^{\circ}, p<0.001\right)$. There was no statistical difference in volar tilt values after 6 months of follow-up. The mean preprocedural radial height was $4.13 \mathrm{~mm}$ $(-7.8 \mathrm{~mm}-9.5 \mathrm{~mm}, \mathrm{SD} \pm 5.06 \mathrm{~mm})$. There was a statistically significant increase at the immediate postoperative period to $10.04 \mathrm{~mm}(p=0.002)$, and a significant reduction at 6 months to $9.55 \mathrm{~mm}(p=0.012)$. The consolidation rate was $100 \%$ with the technique used, with a minimal complication rate. No patient had infection or required a reoperation.

Conclusion The technique was effective for the treatment of distal radial extraarticular fractures at 6 months, with a low complication rate; radiographic parameters values were acceptable and close to the anatomical ones.

\section{Introdução}

As fraturas do rádio distal estão entre as principais lesões de extremidades, compondo $\sim 17 \%$ do total das fraturas de extremidade. ${ }^{1}$ Estima-se que nos Estados Unidos ocorram 640.000 casos anualmente, ${ }^{2}$ embora no Brasil ainda não existam estudos demográficos que evidenciem a real magnitude destas lesões. Acidentes em alta velocidade e a queda de altura estão entre as causas mais frequentes, e os mecanismos mais comuns envolvem o trauma com o punho em hiperextensão ou em flexão. ${ }^{3,4}$

Dentre as opções de tratamento para as fraturas do rádio distal, o tratamento conservador é indicado quando possível a redução da fratura, mantendo-a estável com imobilização gessada, ou quando o paciente não possuir condições clínicas para correção cirúrgica. Já o tratamento operatório é indicado para as fraturas instáveis, intra-articulares e cominutivas. ${ }^{1,4}$

Apesar do avanço e da diversidade de opções dentre as técnicas cirúrgicas nos últimos anos, o tratamento das fraturas da extremidade distal do rádio ainda é assunto controverso. ${ }^{4,5}$ A redução fechada com a fixação de fios de Kirschner percutâneos tem sido o método de tratamento mais comum para fraturas extra-articulares instáveis do rádio distal. ${ }^{4,5}$ No entanto, as limitações e complicações específicas diminuíram sua indicação. A falta de estabilidade, a necessidade de imobilização restritiva, a irritação da pele, a infecção do trajeto dos fios e até lesões nervosas são complicações que podem limitar o sucesso do período de reabilitação pós-operatório. Além disso, até $44 \%$ dos casos podem evoluir com complicações tardias a partir deste procedimento, como migração ou fratura do material, além de consolidação viciosa da fratura. ${ }^{1,6}$

Nos últimos 10 anos, novas opções terapêuticas tecnológicas vêm ganhando destaque. Dentre elas, o tratamento cirúrgico com redução aberta e fixação com placa de bloqueio volar tornou-se um método de tratamento popular para a maioria dos padrões de fratura, pois pode fornecer estabilização rígida, movimentação precoce e recuperação funcional mais rápida. Contudo, apesar das vantagens citadas, esta forma de tratamento apresenta dissecção significativa de tecidos moles, taxas de complicações moderadas e tempo cirúrgico mais longo. ${ }^{7-14}$

No entanto, ainda existem diferentes formas de fixação para fraturas da extremidade distal do rádio, especialmente para as lesões extra-articulares, sem os mesmos riscos de uma cirurgia aberta. A substituição dos fios de Kirschner por parafusos canulados e maciços na cirurgia percutânea tem evidenciado desfechos superiores, menor agressão cirúrgica, menor tempo de imobilização rígida no pós-operatório e menor número de complicações tardias, além de resultados semelhantes aos da placa de bloqueio volar. ${ }^{2-15}$ Diferentemente das outras técnicas percutâneas descritas na literatura, descrevemos uma técnica de fixação utilizando um único parafuso maciço de $3.5 \mathrm{~mm}$ para tratamento de fraturas extra-articulares da extremidade distal do rádio.

\section{Materiais e Métodos}

O presente estudo foi aprovado pelo Comitê de Ética em Pesquisa da instituição sob o número CAAE 00854018.3.0000.5335. Os critérios de inclusão e exclusão estão apresentados na - Tabela 1. Foram incluídos todos os pacientes submetidos à fixação de fratura extra-articular da extremidade distal do rádio com um parafuso maciço de $3.5 \mathrm{~mm}$ no período de outubro de 2016 a dezembro de 2018 . O número total da amostra válida foi de 16 pacientes. Tivemos um paciente excluído por não completar o seguimento mínimo necessário de 6 meses. Foram realizadas avaliações radiográficas no pré-operatório, no pós-operatório imediato e com 6 meses de pós-operatório. Os dados radiográficos coletados foram o tilt voltar (em graus) e a altura radial (em mm).

A análise estatística foi realizada utilizando o software PASW Statistics for Windows, Versão 17 (SPSS Inc. Chicago, IL, EUA), sendo realizado o teste de Friedman com correção de 
Tabela 1 Critérios de Inclusão e Exclusão

\begin{tabular}{|l|l|}
\hline $\begin{array}{l}\text { Critérios de } \\
\text { Inclusão }\end{array}$ & Critérios de Exclusão \\
\hline $\begin{array}{l}\text { Idade entre } \\
18 \text { e } 60 \text { anos }\end{array}$ & $\begin{array}{l}\text { Fraturas complexas extra- e } \\
\text { intra-articulares }\end{array}$ \\
\hline $\begin{array}{l}\text { Boa qualidade } \\
\text { óssea }\end{array}$ & $\begin{array}{l}\text { Lesões operadas com }>14 \text { dias do } \\
\text { trauma inicial }\end{array}$ \\
\hline $\begin{array}{l}\text { Tempo entre } \\
\text { trauma e } \\
\text { cirurgia de } \\
14 \text { dias }\end{array}$ & Cirurgia prévia para mesma fratura \\
\hline & Sinais de osteopenia ou osteoporose \\
\hline & Pacientes com idade $>60$ anos \\
\hline & $\begin{array}{l}\text { Lesões concomitantes em membro } \\
\text { superior ipsilateral }\end{array}$ \\
\hline & Fraturas expostas \\
\hline & Lesões neurovasculares associadas \\
\hline
\end{tabular}

Bonferroni para comparações múltiplas. Para confirmar o achado dos valores, foi realizado o teste de análise de variância (ANOVA, na sigla em inglês) de medidas repetidas com teste post-hoc de Bonferroni

\section{Técnica cirúrgica}

Todos os pacientes apresentavam fraturas extra-articulares do rádio distal (-Figura 1) e foram submetidos ao procedimento no centro cirúrgico. 0 processo anestésico foi realizado com sedação e bloqueio periférico. Após adequada antissepsia, os campos estéreis foram posicionados e um garrote pneumático foi acionado na raiz do membro operado. Após redução incruenta da fratura e alinhamento aceitável atingido através de controle radioscópico, foi realizada incisão única de $1 \mathrm{a} 2 \mathrm{~cm}$ na porção distal do processo estilóide radial ( - Figura 2), entre o primeiro e segundo compartimentos extensores. A dissecção dos tecidos foi realizada até a visualização óssea do ponto de inserção do parafuso. A
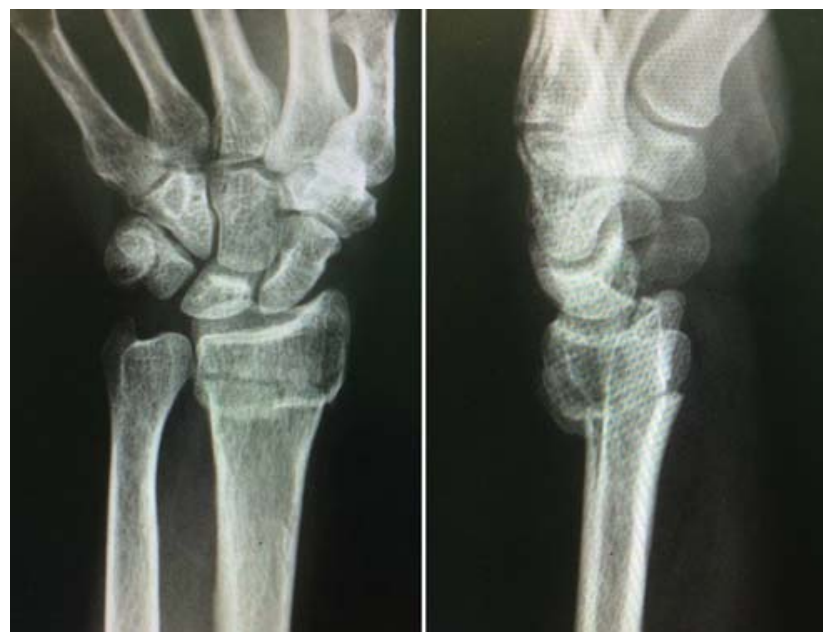

Fig. 1 Radiografias em anteroposterior (AP) e perfil (P) de uma fratura extra-articular da extremidade distal do rádio.

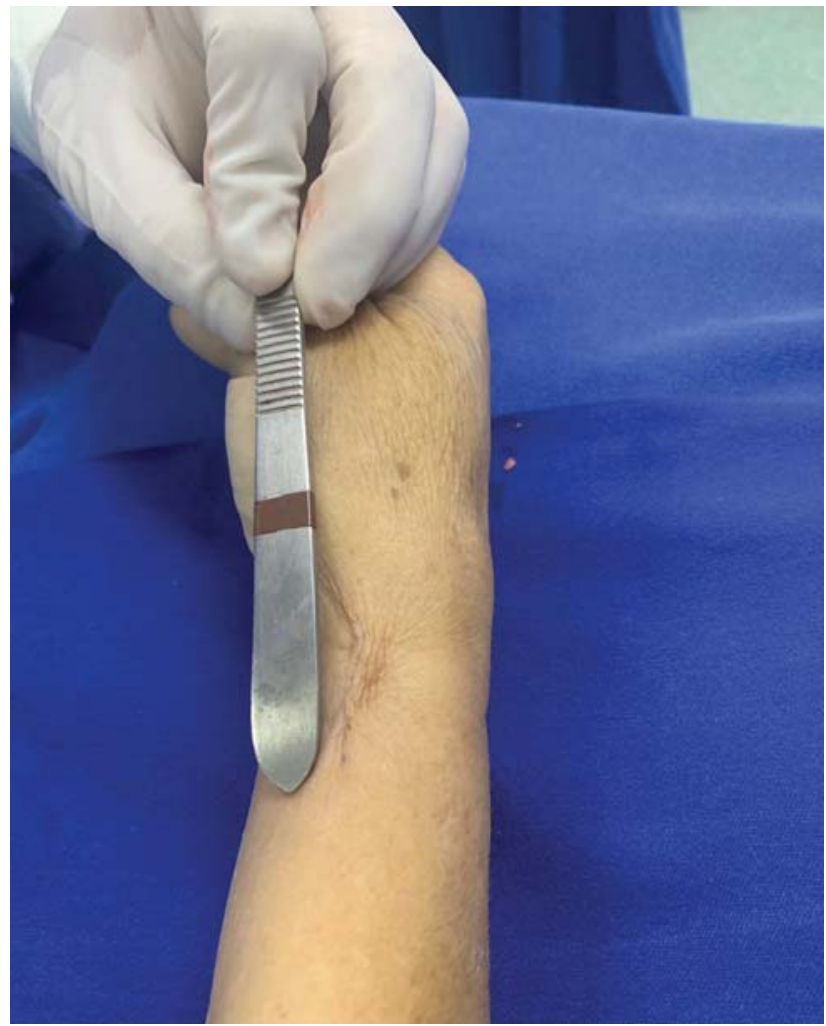

Fig. 2 Incisão para abordagem percutânea da fratura.

perfuração da cortical radial foi realizada com broca $3.5 \mathrm{~mm}$ e da cortical ulnar com broca $2.5 \mathrm{~mm}$, utilizando protetor de partes moles e cuidados para não ocorrer lesões das estruturas neurovasculares desta topografia. O trajeto da broca foi confirmado através de fluoroscopia.

Após a medição e o preparo adequados, foi inserido um parafuso maciço de $3.5 \mathrm{~mm}$ até a cortical medial do rádio. 0 posicionamento do parafuso e a estabilidade da fratura foram checados com fluoroscopia (- Figura 3). Foi realizado fechamento por planos e curativo estéril. Os pacientes foram mantidos com tala antebraquiopalmar por 14 dias.
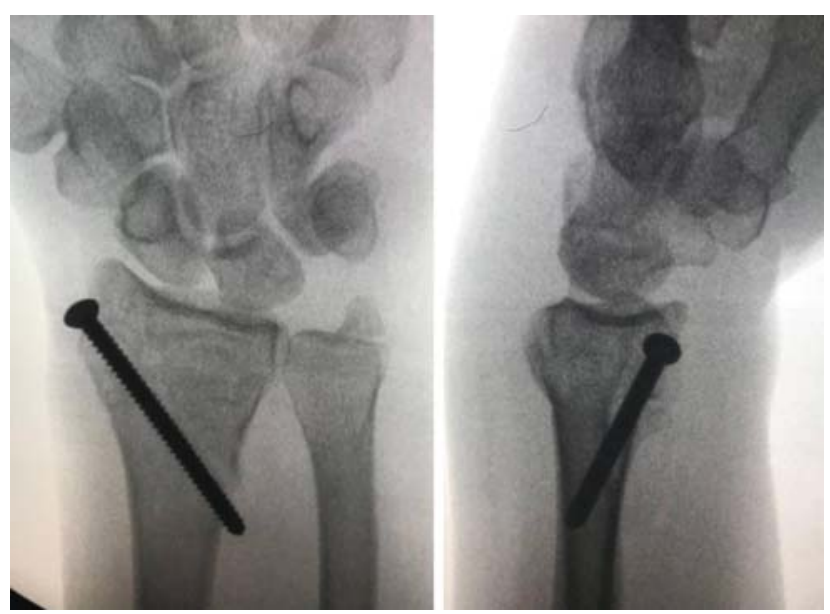

Fig. 3 Controle fluoroscópico da redução e alinhamento da fratura após a fixação com parafuso maciço. 

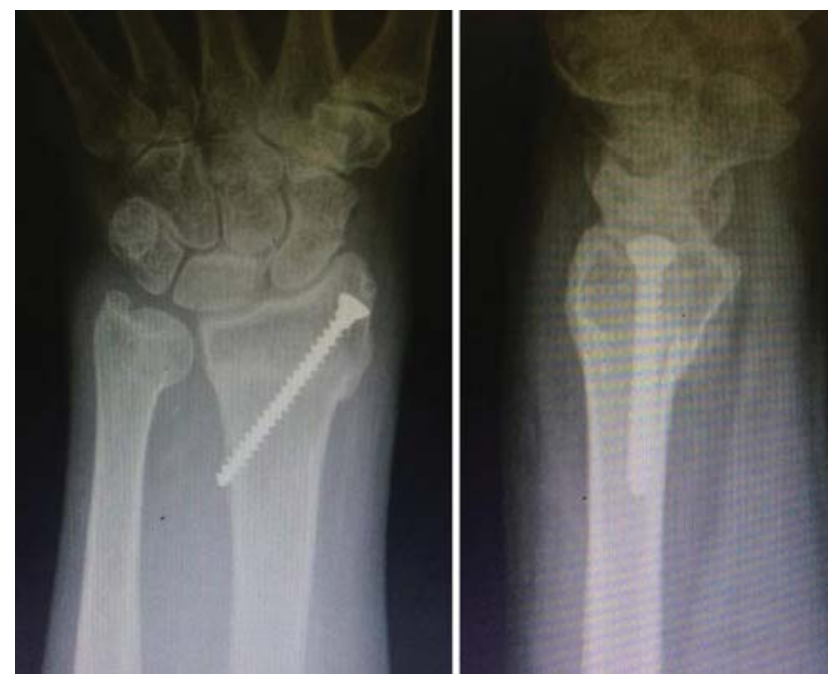

Fig. 4 Radiografias em anteroposterior (AP) e perfil (P) de controle ambulatorial pós-operatório de uma fratura com evidência de consolidação tratada com a técnica apresentada.

\section{Seguimento e pós-operatório}

Após 14 dias de imobilização, os pontos da ferida operatória foram retirados e os pacientes foram liberados para iniciar fisioterapia motora para ganho de amplitude de movimento, analgesia e controle do edema local. O controle radiográfico foi realizado nos retornos ambulatoriais (-Figura 4 ).

\section{Resultados}

Os dados demográficos do estudo estão na - Tabela 2. 0 grupo de estudo inicial incluiu 16 pacientes, (81,25\% mulheres), com média de idade 46,5 anos (25-60 anos). 0 tempo de espera entre a data do trauma até a cirurgia foi de 8,8 dias (4-14 dias). O principal mecanismo de trauma foi queda da própria altura (75\% casos; $n=13$ ), seguido de trauma de alturas maiores ou iguais a 1 metro $(n=2)$ e em vigência de atividade esportiva $(n=1)$.

Os parâmetros radiográficos e análises do tilt Volar estão presentes na - Tabela 3. O tilt Volar médio pré-procedimento foi de $-7,41^{\circ}\left(-23,48^{\circ}-5,29^{\circ}\right.$, desvio padrão (DP) $\left.\pm 6,59^{\circ}\right)$. 0 valor médio imediatamente após a redução e fixação da fratura foi de $5,93^{\circ}\left(\mathrm{DP} \pm 6,23^{\circ}, p<0,001\right)$. Ao final de 6 meses, o valor final médio reduziu para $5,68^{\circ}$, porém esta redução não obteve valores significativamente estatísticos $(p=0,095)$.

Tabela 2 Dados Demográficos

\begin{tabular}{|l|l|}
\hline Amostra $(\boldsymbol{n})$ & 16 pacientes \\
\hline Idade & 46,5 anos $(25-60)$ \\
\hline Gênero & Feminino $n=13 / /$ Masculino $n=3$ \\
\hline $\begin{array}{l}\text { Tempo até o } \\
\text { procedimento (dias) }\end{array}$ & $8,8(4-14$ dias) \\
\hline $\begin{array}{l}\text { Mecanismo } \\
\text { do Trauma }\end{array}$ & $\begin{array}{l}\text { Queda da própria altura }(n=13), \\
\text { queda de altura maior ou igual } \\
\text { a 1 metro }(n=2), \text { atividade } \\
\text { esportiva }(n=1)\end{array}$ \\
\hline
\end{tabular}

Tabela 3 Comparações entre as mensurações do tilt volar (em graus) nos 3 períodos do estudo

\begin{tabular}{|c|c|c|c|}
\hline & $\begin{array}{l}\text { Tilt volar } \\
\text { Pré-operatório }\end{array}$ & $\begin{array}{l}\text { Tilt Volar } \\
\text { Pós- operatório } \\
\text { Imediato }\end{array}$ & valor-p \\
\hline Média & $\begin{array}{l}-7,41 \\
(-23,48-5,29)\end{array}$ & $\begin{array}{l}5,94 \\
(-11,46-12,00)\end{array}$ & $<\underline{0,001}$ \\
\hline \multirow{2}{*}{$\begin{array}{l}\text { Desvio } \\
\text { Padrão }\end{array}$} & $\pm 6,59$ & $\pm 6,24$ & - \\
\hline & Tilt volar Pré & $\begin{array}{l}\text { Tilt Volar } \\
6 \text { meses }\end{array}$ & $p$ \\
\hline Média & $\begin{array}{l}-7,41 \\
(-23,48-5,29)\end{array}$ & $\begin{array}{l}5,68 \\
(-11,40-12,00)\end{array}$ & $<\underline{0,001}$ \\
\hline \multirow{2}{*}{$\begin{array}{l}\text { Desvio } \\
\text { Padrão }\end{array}$} & $\pm 6,59$ & $\pm 6,27$ & - \\
\hline & $\begin{array}{l}\text { Tilt volar } \\
\text { Pós - Imediato }\end{array}$ & $\begin{array}{l}\text { Tilt Volar } \\
6 \text { meses }\end{array}$ & $p$ \\
\hline Média & $\begin{array}{l}5,94 \\
(-11,46-12,00)\end{array}$ & $\begin{array}{l}5,68 \\
(-11,40-12,00)\end{array}$ & 0,095 \\
\hline $\begin{array}{l}\text { Desvio } \\
\text { Padrão }\end{array}$ & $\pm 6,24$ & $\pm 6,27$ & \\
\hline
\end{tabular}

Os parâmetros radiográficos e análises da altura radial estão presentes na - Tabela 4 . A média da altura radial pré-operatória foi de $4,13 \mathrm{~mm}\left(-7,8^{\circ}-9,5^{\circ}, \mathrm{DP} \pm 5,06^{\circ}\right)$. 0 valor médio da altura radial no pós-operatório imediato foi $10,04 \mathrm{~mm}$, com aumento estatisticamente significativo $(p=0,002)$. Ao final de 6 meses, o valor médio reduziu para $9,55 \mathrm{~mm}$, com redução também estatisticamente significativa entre os 2 períodos $(p=0,012)$

Em 3 meses de pós-operatório, obtivemos consolidação de $100 \%$ das fraturas (-Figuras 5,6 e $\mathbf{7}$ ), e todos os pacientes

Tabela 4 Comparações entre as mensurações da Altura Radial (em $\mathrm{mm}$ ) nos 3 períodos do estudo

\begin{tabular}{|l|l|l|l|}
\hline & $\begin{array}{l}\text { Altura } \\
\text { Radial Pré }\end{array}$ & $\begin{array}{l}\text { Altura } \\
\text { Radial Pós - } \\
\text { Imediato }\end{array}$ & $p$ \\
\hline Média & $\begin{array}{l}4,13 \\
(-7,8-9,5)\end{array}$ & $\begin{array}{l}10,04 \\
(+6,6-11,30)\end{array}$ & $\underline{\mathbf{0 , 0 0 2}}$ \\
\hline $\begin{array}{l}\text { Desvio } \\
\text { Padrão }\end{array}$ & $\pm 5,06$ & $\pm 1,26$ & - \\
\hline & $\begin{array}{l}\text { Altura } \\
\text { Radial Pré }\end{array}$ & $\begin{array}{l}\text { Altura } \\
\text { Radial 6 meses }\end{array}$ & $\boldsymbol{p}$ \\
\hline Média & $\begin{array}{l}4,13 \\
(-7,8-9,5)\end{array}$ & $\begin{array}{l}9,55 \\
(+5,70-11,00)\end{array}$ & $\underline{\mathbf{0 , 0 0 7}}$ \\
\hline $\begin{array}{l}\text { Desvio } \\
\text { Padrão }\end{array}$ & $\begin{array}{l} \pm 5,06 \\
\text { Altura } \\
\text { Radial Pós - } \\
\text { Imediato }\end{array}$ & $\begin{array}{l}\text { Altura } \\
\text { Radial 6 meses }\end{array}$ & $\boldsymbol{p}$ \\
\hline $\begin{array}{l}\text { Média } \\
(+6,04\end{array}$ & $\begin{array}{l}9,55 \\
(+5,70-11,00)\end{array}$ & $\underline{\mathbf{0 , 0 1 2}}$ \\
\hline $\begin{array}{l}\text { Desvio } \\
\text { Padrão }\end{array}$ & $\pm 1,26$ & $\pm 1,51$ & \\
\hline
\end{tabular}



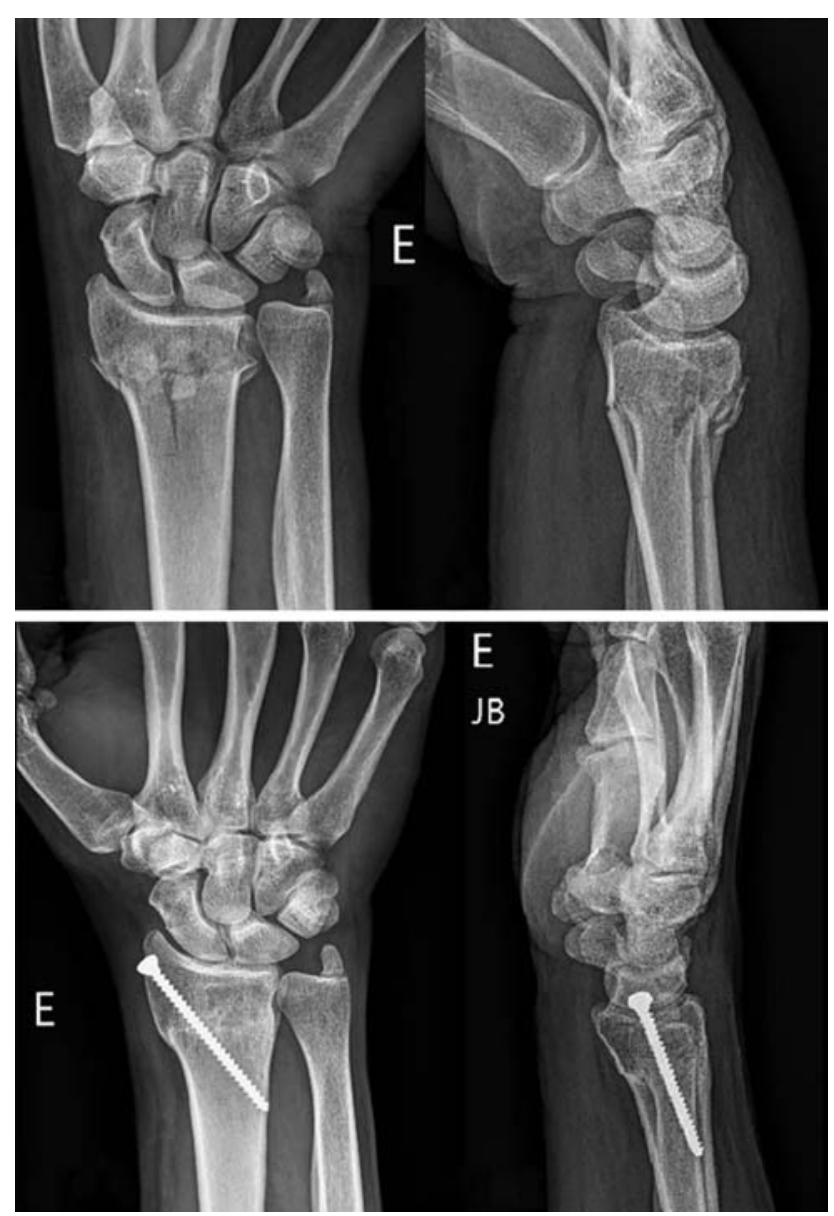

Fig. 5 Sequência de radiografias pré- e pós-operatórias de fraturas tratadas com a técnica apresentada.

apresentavam amplitude de movimento satisfatória (- Figura 8). Um paciente operado com esta técnica permaneceu com dor crônica no pós-operatório, muito provavelmente causada pela pseudoartrose do processo estilóide da ulna. Não apresentamos quadros infecciosos e nenhum paciente precisou ser reoperado.

Ao final de 6 meses, obtivemos parâmetros radiográficos próximos dos valores anatômicos. Um paciente apresentou resultado radiográfico insatisfatório, com tilt volar de $-11,4^{\circ}$. Creditamos este resultado à falha técnica durante a redução da fratura deste paciente.

\section{Discussão}

O interesse pela osteossíntese minimamente invasiva tem crescido em todo o mundo. ${ }^{16} \mathrm{O}$ desenvolvimento dos dispositivos de imagem e de fixação possibilitaram aos cirurgiões ortopédicos tratarem diferentes tipos de fraturas com menores dissecção e agressão cirúrgica. Pacientes submetidos a cirurgias minimamente invasivas tendem a apresentar menores incisões, mínima perda de sangue, menor tempo de internação e retorno mais rápido às atividades diárias. ${ }^{17}$

Apesar de historicamente a fixação percutânea com fios de Kirschner ter sido amplamente utilizada para tratamento de fraturas extra-articulares do rádio distal, a tendência da utilização das placas de bloqueio volar é cada vez maior. A
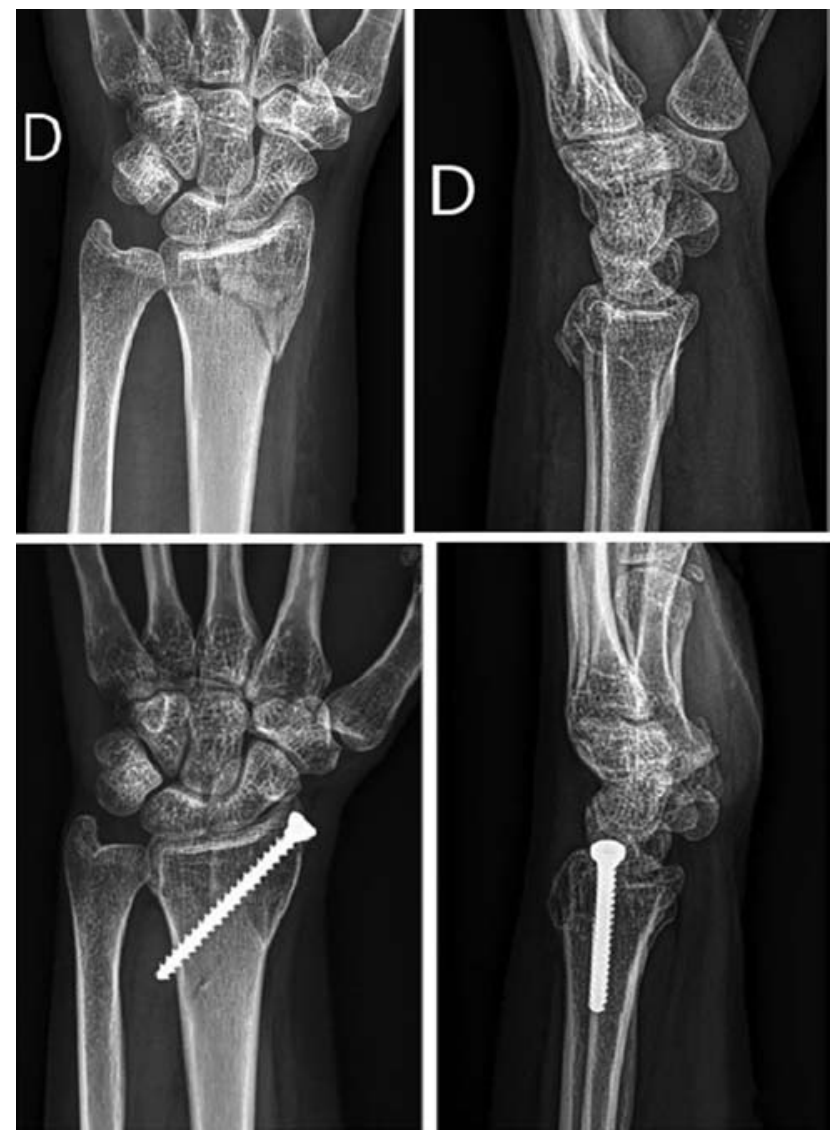

Fig. 6 Sequência de radiografias pré- e pós-operatórias de fraturas tratadas com a técnica apresentada.

falta de estabilidade da fixação com os fios, somada às chances de consolidação viciosa e infecção do trajeto dos pinos são algumas das principais razões que estão levando esta técnica a perder popularidade.

Um estudo realizado por Gereli et al. ${ }^{15}$ comparou os resultados entre a fixação percutânea com dois parafusos canulados e a fixação com placa de bloqueio volar. Em relação ao tempo cirúrgico, o grupo com parafusos canulados teve duração média de procedimento de 53 minutos (40-80 minutos) comparados com 77,7 minutos no grupo da fixação com placa de bloqueio volar (60-90 minutos), com $p=0.001$. Em relação à amplitude de movimento, no acompanhamento de 2 meses, os pacientes do grupo da fixação percutânea apresentaram maiores amplitudes de movimento na pronação $\left(66^{\circ}\right.$ versus $58^{\circ}$, $p=0.005)$ e na supinação $\left(59^{\circ} \times 51^{\circ}, p=0.025\right)$, porém, ao final do estudo, ambos os grupos apresentavam amplitudes de movimento iguais. $\mathrm{O}$ estudo ainda sugeriu que não há diferença significativa entre os grupos no que tange o retorno precoce às atividades diárias.

Uma recente metanálise ${ }^{17}$ comparando tratamento com redução aberta e fixação interna (RAFI) versus fixação externa mostrou resultados significativamente superiores no questionário Disabilities of the Arm, Shoulder and Hand (DASH, na sigla em inglês) em favor do grupo RAFI utilizando placas de bloqueio volar. $O$ questionário avalia a capacidade do paciente de executar suas atividades diárias utilizando o membro superior e configura um importante método 

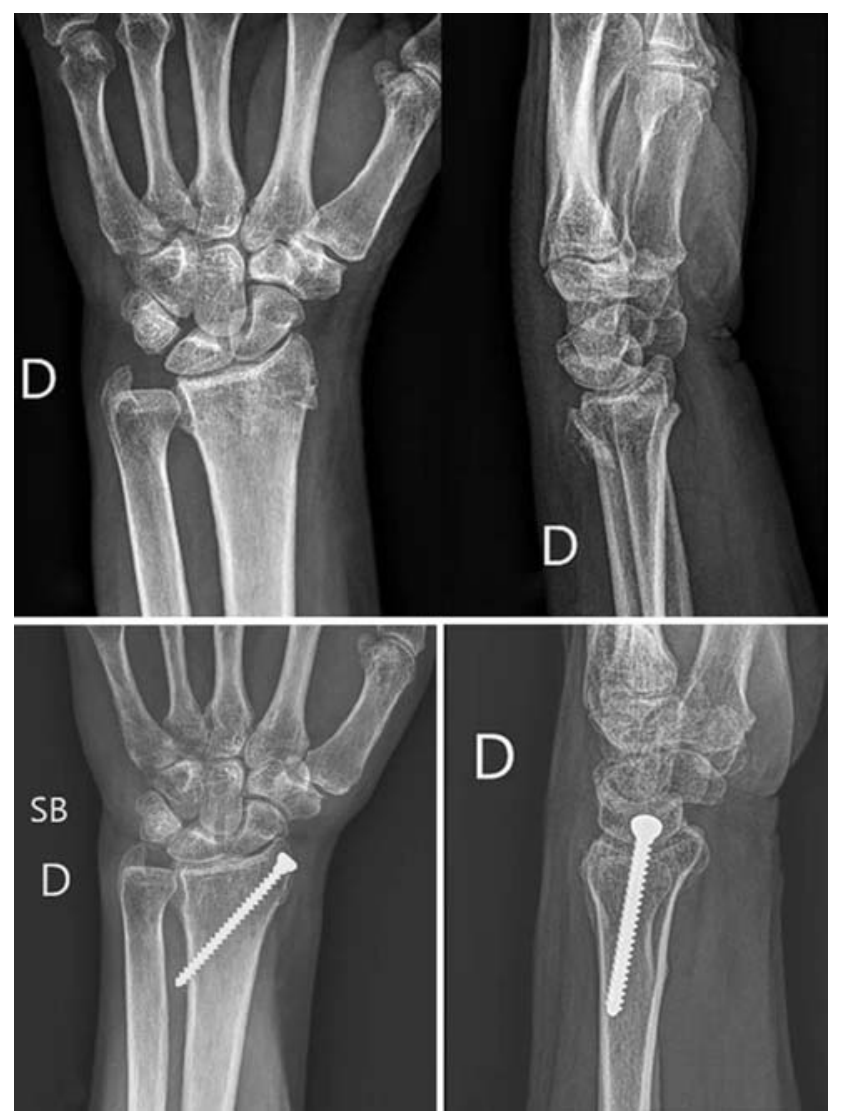

Fig. 7 Sequência de radiografias pré- e pós-operatórias de fraturas tratadas com a técnica apresentada.

validado para avaliação funcional pós-operatória. Além disso, o estudo ainda conclui que o grupo RAFI possui melhor restauração do tilt volar e melhor supinação do antebraço, porém força de preensão menor quando comparado com a fixação externa.

Jupiter et al. ${ }^{19}$ e Zhang et al..$^{20}$ discutem que a mobilidade precoce que a placa de bloqueio volar permite seria um dos principais fatores para uma melhora clínica significativa. Contudo, Handoll et al., ${ }^{21}$ em revisão sistemática pelo banco de dados Cochrane, concluiu que a evidência dos estudos rando- mizados em reabilitação de fraturas do rádio distal em adultos é insuficiente para estabelecer a efetividade entre o tempo de imobilização e as diferentes intervenções no pós-operatório. Handoll et al. ${ }^{21}$ sugerem, ainda, que os grandes centros devem identificar questões prioritárias na reabilitação dos pacientes para melhorar a qualidade da evidência científica produzida.

Stinton et al. ${ }^{22}$ realizaram uma metanálise longitudinal de última geração para avaliar a recuperação durante os 2 primeiros anos pós-operatórios após uma fratura de rádio distal tratada com placa de bloqueio volar. Os autores concluíram que a melhora clínica significativa pode ser atingida em até 12 meses, atingindo após esse período um "platô estável" e níveis de normalidade prévia após a cirurgia.

Revisando estudos na literatura nacional, Pardini Júnior et al. ${ }^{23}$ avaliaram os resultados radiográficos de 34 pacientes submetidos ao tratamento percutâneo das fraturas da extremidade distal do rádio com fios de Kirschner. Apesar de terem apresentado resultados com piora média do tilt volar da fratura $\left(-2,03^{\circ}\right.$ no período pós-operatório imediato versus - 3,82 ${ }^{\circ}$ ao final do estudo), não foi encontrada significância estatística $(p=0,511)$.

Piva Neto et al. ${ }^{24}$ também analisaram radiograficamente os resultados do tratamento percutâneo ao final de 6 semanas. Este estudo não comparou os parâmetros radiográficos do pósoperatório imediato com os parâmetros ao final do período avaliado, fato que impede de avaliar uma possível perda de redução da fratura ao longo do processo de consolidação.

Comparando o presente estudo com os trabalhos nacionais citados anteriormente, obtivemos resultados superiores em relação à restauração do tilt volar médio $\left(5,68^{\circ}\right.$ em comparação a $-3,82^{\circ}$ e $3,3^{\circ}$ ) e resultados similares na restauração da altura radial comparado ao estudo de Piva Neto et al. ${ }^{24}$ (9,55 $\mathrm{mm}$ em comparação a 9,47 mm). Além disso, a técnica utilizada apresentou um menor número de complicações e nenhum paciente apresentou quadro infeccioso.

A técnica apresentada no presente artigo pode ser utilizada como uma opção de baixo custo para o tratamento das fraturas extra-articulares do rádio distal. Países com menor poder aquisitivo precisam ter alternativas para contornar a baixa disponibilidade de placas de bloqueio volar.

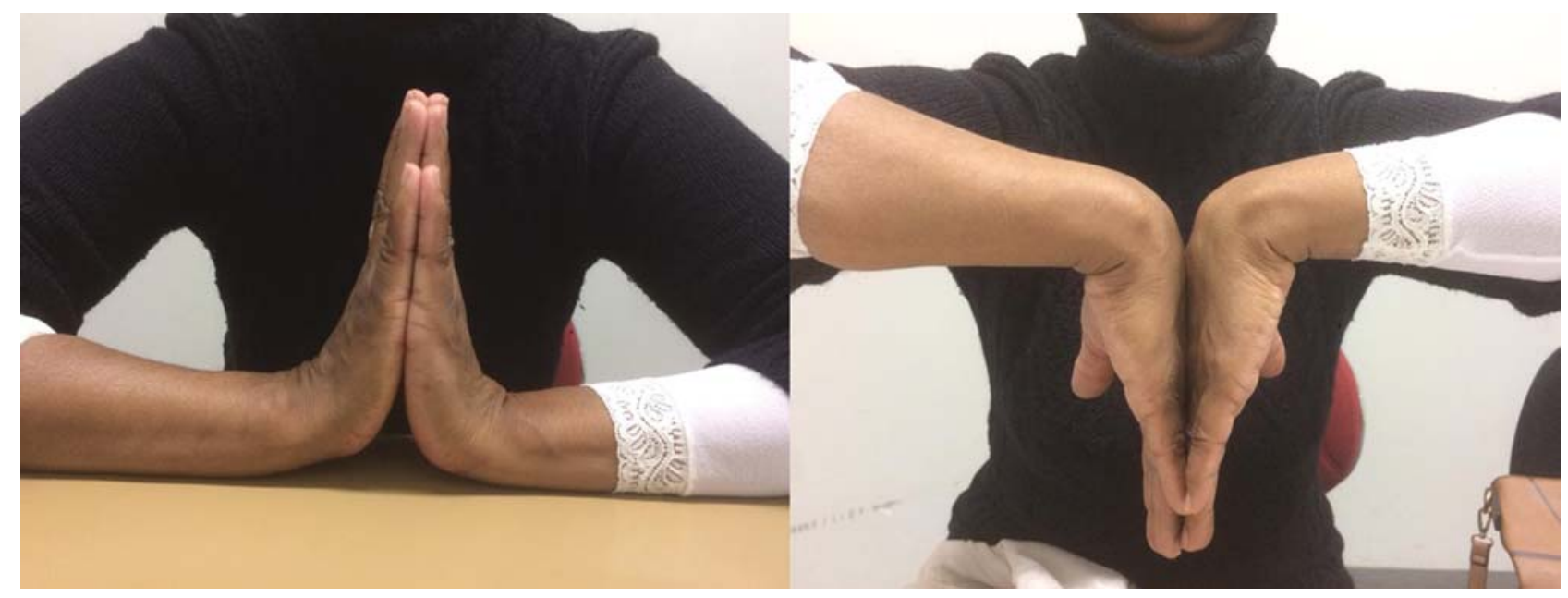

Fig. 8 Amplitude de movimento seis meses após a cirurgia. 
A fixação percutânea das fraturas com um parafuso maciço de $3.5 \mathrm{~mm}$ permitiu uma fixação estável das fraturas e início precoce de fisioterapia motora, mantendo os parâmetros radiográficos similares aos valores anatômicos ao final do período de 6 meses. Além disso, a mínima incisão realizada para a osteossíntese e a mínima dissecção de partes moles também proporcionaram dor mínima no pós-operatório imediato. Não apresentamos casos de infecção durante todo seguimento.

O presente estudo possui algumas limitações. O tipo específico de fratura incluído no trabalho, o tipo de fixação utilizado e os diversos critérios de inclusão e exclusão restringem significativamente o número da amostra e o poder do estudo. Além disso, consideramos elevado o tempo médio entre a data inicial do trauma até o procedimento cirúrgico (média de 8,8 dias). Por se tratar de um estudo realizado no sistema público de saúde, muitas vezes a burocracia e a hierarquização do sistema podem levar a um maior tempo de espera, fator que pode estar relacionado diretamente a resultados inferiores. Outra limitação foi a impossibilidade de padronizar os protocolos de fisioterapia motora no pós-operatório.

\section{Conclusão}

A fixação percutânea com parafuso cortical de $3.5 \mathrm{~mm}$ é uma opção útil e de baixo custo para o tratamento das fraturas extra-articulares da extremidade distal do rádio. A técnica mostrou-se bastante efetiva, com manutenção aceitável dos parâmetros radiográficos, permitindo mobilização precoce do punho e um baixo índice de complicações.

\section{Conflito de Interesses}

Os autores declaram não haver conflito de interesses.

\section{Referências}

1 Jakubietz MG, Gruenert JG, Jakubietz RG. Palmar and dorsal fixedangle plates in AO C-type fractures of the distal radius: is there an advantage of palmar plates in the long term? J Orthop Surg Res 2012;7(01):8

2 Taras JS, Saillant JC, Goljan P, McCabe LA. Distal Radius Fracture Fixation With the Specialized Threaded Pin Device. Orthopedics 2016;39(01):e98-e103

3 Clé P, Tasso L, Barbosa R, et al. Estudo retrospectivo do estado funcional de pacientes com fratura do rádio distal submetidos à osteossíntese com placa LCP. Acta Fisiátrica 2011;18(04):163-168

4 Fernandez DL, Wolfe SW. Distal radius fractures. In: Green DP, Hotchkiss RN, Pederson WC, Scott WW, editors. Green's operative hand surgery. Philadelphia: Churchill Livingstone; 2005:656

5 Tang JB. Distal radius fracture: diagnosis, treatment, and controversies. Clin Plast Surg 2014;41(03):481-499

6 Taras JS, Ladd AL, Kalainov DM, Ruch DS, Ring DC. New concepts in the treatment of distal radius fractures. Instr Course Lect 2010; 59:313-332
7 Hsu LP, Schwartz EG, Kalainov DM, Chen F, Makowiec RL. Complications of K-wire fixation in procedures involving the hand and wrist. J Hand Surg Am 2011;36(04):610-616

8 Rozental TD, Blazar PE. Functional outcome and complications after volar plating for dorsally displaced, unstable fractures of the distal radius. J Hand Surg Am 2006;31(03):359-365

9 Orbay JL, Fernandez DL. Volar fixation for dorsally displaced fractures of the distal radius: a preliminary report. J Hand Surg Am 2002;27(02):205-215

10 Lichtman DM, Bindra RR, Boyer MI, et al. Treatment of distal radius fractures. J Am Acad Orthop Surg 2010;18(03):180-189

11 Arora R, Lutz M, Hennerbichler A, Krappinger D, Espen D, Gabl M. Complications following internal fixation of unstable distal radius fracture with a palmar locking-plate. J Orthop Trauma 2007;21 (05):316-322

12 Shyamalan G, Theokli C, Pearse Y, Tennent D. Volar locking plates versus Kirschner wires for distal radial fractures-a cost analysis study. Injury 2009;40(12):1279-1281

13 Koval KJ, Harrast JJ, Anglen JO, Weinstein JN. Fractures of the distal part of the radius. The evolution of practice over time. Where's the evidence? J Bone Joint Surg Am 2008;90(09):1855-1861

14 Prommersberger KJ, Pillukat T, Mühldorfer M, van Schoonhoven J. Malunion of the distal radius. Arch Orthop Trauma Surg 2012;132 (05):693-702

15 Gereli A, Nalbantoglu U, Kocaoglu B, Turkmen M. Comparative study of the closed reduction percutaneous cannulated screw fixation and open reduction palmar locking plate fixation in the treatment of AO type A2 distal radius fractures. Arch Orthop Trauma Surg 2014;134(01):121-129

16 Nalbantoglu U, Gereli A, Kocaoglu B, Turkmen M. Percutaneous cannulated screw fixation in the treatment of distal radius fractures. Arch Orthop Trauma Surg 2012;132(09):1335-1341

17 Yeung SH. Minimally invasive surgery in orthopaedics. Small is beautiful? Hong Kong Med J 2008;14(04):303-307

18 Wei DH, Poolman RW, Bhandari M, Wolfe VM, Rosenwasser MP. External fixation versus internal fixation for unstable distal radius fractures: a systematic review and meta-analysis of comparative clinical trials. J Orthop Trauma 2012;26(07):386-394

19 Jupiter JB, Marent-Huber M; LCP Study Group. Operative management of distal radial fractures with 2.4-millimeter locking plates. A multicenter prospective case series. J Bone Joint Surg Am 2009;91(01):55-65

20 Zhang Q Liu F, Xiao Z, et al. Internal Versus External Fixation for the Treatment of Distal Radial Fractures: A Systematic Review of Overlapping Meta-Analyses. Medicine (Baltimore) 2016;95(09): e2945

21 Handoll HH, Elliott J. Rehabilitation for distal radial fractures in adults. Cochrane Database Syst Rev 2015;(09):CD003324

22 Stinton SB, Graham PL, Moloney NA, Maclachlan LR, Edgar DW, Pappas E. Longitudinal recovery following distal radial fractures managed with volar plate fixation. Bone Joint J 2017;99-B(12): 1665-1676

23 Pardini AG Junior, Bufaical HGF, Freitas AD, Chaves AB. Resultados funcionais e radiológicos a longo prazo da fixação percutânea das fraturas da extremidade distal do rádio. Rev Bras Ortop 2012;47 (01):31-36

24 Piva Neto A, Lhamby FC. Fixação das fraturas da extremidade distal do rádio pela técnica de kapandji modificada: avaliação dos resultados radiológicos. Rev Bras Ortop 2011;46(04):368-373 